\title{
Pedagogía social
}

Social education / Pedagogia social

La excelencia en los doctorados en educación: Brasil, México y Argentina

Excellent Doctorates in Education: Brazil, Mexico and Argentina

A excelência nos doutorados em educação: Brasil, México e Argentina

Luis Sime-Poma, Pontificia Universidad Católica del Perú, Perú 



\section{La excelencia en los doctorados en educación: Brasil, México y Argentina}

\section{Luis Sime-Poma}

Pontificia Universidad Católica del Perú, Perú

lsime@pucp.pe

\section{Resumen}

A través de este estudio exploratorio se identifican los programas de Doctorado en Educación considerados de excelencia por los sistemas públicos de acreditación de Brasil, México y Argentina. El estudio describe y compara las líneas de investigación de dichos programas, algunos rasgos de sus docentes investigadores (cantidad, nivel de acreditación como investigadores, cantidad y características de artículos publicados). Para ello, se utiliza el análisis documental de la información oficial brindada por los doctorados y las agencias públicas concernidas en sus respectivas páginas electrónicas.

El estudio revela datos significativos sobre los doctorados aunque desiguales en la información aportada en sus páginas electrónicas y muestra cómo estos programas contribuyen a la legitimidad científica de la educación, como área de conocimiento relevante ante la cual es posible organizar comunidades académicas comprometidas con la producción multidisciplinaria de conocimientos y formación de investigadores para dicha área tan reconocida como otras comunidades científicas universitarias.

\section{Palabras clave}

Grado de doctor, formación de investigadores, investigación pedagógica, personal académico docente, universidad, educación. (Fuente: Tesauro de la Unesco).

Recepción: 2013-07-15 | Envío a pares: 2013-09-29 | Aceptación por pares: 2013-10-14 | Aprobación: 2013-11-04 Para citar este artículo / To reference this article / Para citar este artigo 


\title{
Excellent Doctorates in Education: Brazil, Mexico and Argentina
}

\begin{abstract}
The doctoral programs in education regarded as excellent by the public systems for accreditation in Brazil, Mexico and Argentina are identified through this exploratory study. Specifically, it describes and compares the lines of research pursued by those programs, as well as some of the characteristics of the participating teachers-researchers (quantity, extent of accreditation as researchers, quantity and characteristics of articles published). The findings are based on a documentary analysis of official information about doctoral programs and the public agencies involved, as provided on their websites.

The study reveals significant data on these programs, although the information on the websites varies. It also shows they contribute to the scientific legitimacy of education as a relevant field of knowledge around which it is possible to organize academic communities that are committed to generating multidisciplinary knowledge and to training researchers in this area, which is recognized the same as other university scientific communities.
\end{abstract}

\section{Key Words}

Doctoral degrees, research training, educational research, academic teaching personnel, university, education. (Source: UNESCO Thesaurus). 


\section{A excelência nos doutorados em educação: Brasil, México e Argentina}

\section{Resumo}

Por meio deste estudo exploratório, identificam-se os programas de doutorado em educação considerados de excelência pelos sistemas públicos de acreditação do Brasil, México e Argentina. Este estudo descreve e compara as linhas de pesquisa desses programas, alguns traços de seus docentes pesquisadores (quantidade, nível de acreditação como pesquisadores, quantidade e características dos artigos publicados). Para isso, utiliza-se a análise documental da informação oficial oferecida pelos doutorados e pelas agências públicas referenciadas em suas respectivas páginas eletrônicas. O estudo revela dados significativos sobre os doutorados, embora desiguais na informação dada em suas páginas eletrônicas, e mostra como esses programas contribuem para a legitimação científica da educação como área de conhecimento relevante, ante a qual é possivel organizar comunidades acadêmicas comprometidas com a produção multidisciplinar de conhecimentos e formação de pesquisadores para essa área tão reconhecida como outras comunidades cientificas universitárias.

\section{Palavras-chave}

Doutorado, formação em investigação, a pesquisa educacional, pessoal docente acadêmicos, universidade, educação. (Fonte: Tesauro da UNESCO). 


\section{Introducción}

Las dificultades que atraviesan los sistemas educativos no solo son problemas de mejoramiento del capital físico sino también del capital intelectual, y este, a diferencia del primero, requiere otras políticas para potenciar las capacidades humanas; una de ellas es la capacidad para problematizar las prácticas educativas y los procesos macrosociales que la condicionan, cuestión que demanda una experiencia formativa propia de los posgrados. En ese sentido, los programas de posgrado en educación juegan un papel importante en la producción y circulación de conocimientos nacionales e internacionales para la comunidad académica, los docentes y los decisores de políticas públicas. Especialmente, los programas de doctorado en educación son cruciales en la medida en que es en ese nivel de formación donde se acreditan académicamente los nuevos investigadores educacionales y es uno de los lugares clave desde donde se producen conocimientos sistemáticos y rigurosos para el campo educativo.

El crecimiento de las maestrías en educación, la exigencia en diferentes universidades de contar con más doctores para mejorar sus acreditaciones, así como los estímulos a los profesionales del sector educativo con grados académicos más altos y la propia competencia entre programas de posgrado, han generado un mayor interés hacia el doctorado, en un proceso donde la oferta no solo es pública sino también privada y, en donde, además, no solo es ofertado por instituciones nacionales sino también internacionales, en modalidades presenciales y no presenciales. En general, estamos ante una tendencia de "postgraduarización" (Rama, 2006), que se ha flexibilizado, pluralizado y globalizado. En efecto, esta mayor interacción de oferta y demanda de doctorados es parte de una tendencia en diversos países de la región; así, Brasil graduaba en 1980 el equivalente a $3 \%$ de los doctores que graduaba Estados Unidos, en 2005 aumentó a $25 \%$, situándose entre los diez primeros países del mundo de mayor graduación de doctores (Viotti, citado por Gusmão,
2010). Por otro lado, Brasil, México y Argentina ocupan los mayores porcentajes de programas de doctorado en América Latina con una distancia significativa respecto a los demás países (ver la Tabla 1).

Tabla 1. Doctorados en América Latina

\begin{tabular}{|c|c|c|c|}
\hline País & Doctorados & $\%$ & $\begin{array}{c}\text { Universidades } \\
\text { con programas }\end{array}$ \\
\hline Brasil & 1.056 & $48.3 \%$ & 52 \\
\hline México & 406 & $18.5 \%$ & 80 \\
\hline Argentina & 291 & $13.3 \%$ & s/d \\
\hline Chile & 103 & $4.7 \%$ & 10 \\
\hline Cuba & 95 & $4.3 \%$ & 12 \\
\hline Perú & 91 & $4.2 \%$ & 17 \\
\hline Colombia & 56 & $2.6 \%$ & 17 \\
\hline Venezuela & 48 & $2.2 \%$ & 7 \\
\hline Uruguay & 11 & $0.5 \%$ & 1 \\
\hline Ecuador & 9 & $0.4 \%$ & 4 \\
\hline Bolivia & 8 & $0.4 \%$ & 5 \\
\hline Paraguay & 7 & $0.3 \%$ & 4 \\
\hline Panamá & 5 & $0.2 \%$ & 4 \\
\hline Total & 2.188 & $100 \%$ & 213 \\
\hline
\end{tabular}

Fuente: Convenio André Bello e IESALC (2003-2004), en Rama (2006, p. 48)

La literatura sobre doctorados en educación de América Latina no siempre es fácil de identificar debido a que parte de esta se halla incluida en títulos que aluden en general a los posgrados en educación; no obstante, entre las fuentes que sí explicitan en sus títulos y resúmenes el doctorado en educación como objeto de estudio encontramos al menos dos segmentos en función de sus contextos de referencia. En el primer segmento se sitúan los estudios sobre un programa de doctorado de una o varias institución específicas de un país, vinculada o no a un área de especialización particular, sea para describir y analizar sus condiciones institucionales, percepciones de sus docentes y/o alumnos sobre algún aspecto del programa o tipos de impactos de dicha formación (Torres, 2011; Moreno, 2007; Acuña, 2009; Zaidan, et al., 2011; Duarte, 2010; Gutiérrez y Barrón, 2008; Martínez, 2001); mientras que el segundo segmento se diferencia del anterior en la medida 
en que aporta comparaciones entre programas de doctorado de varios países de América Latina. Esta última está menos desarrollada y es menos visible en las bases de datos internacionales, así en Redaly c y Dialnet con las palabras clave "doctorado educación", en la primera aparecen 144 y en el segundo 150, para ambos casos con ningún artículo de tipo comparativo a nivel de la región; al rastrear con términos más amplios "posgrado" y “educación", en Redaly c, se identifican menos de 20 y en Dialnet 150 fuentes, para ambos casos con el mismo resultado negativo. Parte de estos déficits de investigaciones comparativas pueden atribuirse a la limitada presencia de la educación comparada como disciplina durante la formación docente unido también a la poca presencia y trayectoria de instituciones más especializadas en dicha disciplina como las sociedades de educación comparada en la región. (Fernández, Mollis y Dono, 2005).

Los aportes más comparativos a nivel de posgrados en general los encontramos más bien en las publicaciones promovidas desde el Instituto Internacional para la Educación Superior en América Latina y el Caribe (IESALC) de la UNESCO, con sede en Venezuela; no encontrando en su página web textos específicos sobre doctorados en educación; otros son estudios más ocasionales como los de Marquis, Spagnolo y Valenti (1998), que hace catorce años estudiaban la acreditación en los posgrados de Argentina, México y Brasil.

Creemos que la falta de estudios más comparativos entre países de la región genera un déficit de contextualización en la investigación académica que permita descubrir tendencias y valorar más las peculiaridades cuando estas son puestas en relación con otras. Definitivamente, son mayores las aproximaciones comparativas relacionadas con la educación básica que con la educación superior a nivel de posgrados y mucho menor en el campo de los doctorados. La necesidad de avanzar en la construcción del espacio latinoamericano de educación superior (Fernández, 2012) implica, entre otros aspectos, un mayor conocimiento recíproco de los problemas y avances que se vienen procesando en dicho espacio y avanzar en políticas de integración dado que los programas de postgrado se relacionan más con programas de Europa y América del Norte, que con los de la misma región (Rama, 2006; Zarur, 2008).

\section{Método}

El presente estudio exploratorio de carácter descriptivo y documental se propone investigar los doctorados en educación considerados de excelencia de acuerdo con los criterios y mecanismos establecidos por las instancias públicas pertinentes. Más precisamente este estudio se plantea como objetivos específicos, en primer lugar, identificar los programas de doctorado en educación mejor evaluados en tres países de América Latina: Brasil, México y Argentina. Para ello se analizó la información de los órganos de acreditación nacional de dichos países a fin de localizar los niveles obtenidos por los doctorados en educación y especificar los programas que alcanzaron el mayor nivel.

Un segundo objetivo específico ha estado dirigido al análisis de dos variables sustanciales para explorar programas de posgrado como los doctorados: las líneas de investigación y el perfil de sus docentes. Para lo primero se consideró como indicadores el número las líneas de investigación y el discurso utilizado para su justificación; y para el perfil del docente, se sistematizó información sobre su cantidad y pertenencia al sistema evaluador que en cada país los acredita y clasifica como investigadores; así como el número de artículos publicados en revistas académicas en los últimos cinco años, el número de artículos publicados en coautorías y en idiomas extranjeros. En función de estas finalidades se recolectó la información necesaria en los portales electrónicos institucionales de los programas de posgrado, procesándola a través de tablas comparativas. Cabe advertir las dificultades para obtener una información completa, sobre todo para el caso argentino, que limitó las posibilidades de efectuar comparaciones 
mayores; en ese sentido, mientras para responder al primer objetivo fue posible contar con información de los programas de los tres países, para el segundo se contó con datos de los programas de dos países (México y Brasil).

\section{Resultados}

En América Latina las políticas de regulación para evaluar y acreditar la calidad del servicio que ofrecen los posgrados han sido heterogénea; por un lado, podemos advertir países con mayores regulaciones estatales, como es el caso de Brasil, México y Argentina (Villanueva, 2008; Cardoso y Cerecedo, 2011; Santos y Azevedo, 2009) y, por otro lado, están países donde dicha regulación ha sido mínima, como en el caso peruano. Respecto a los primeros, se distinguen dos tipos de experiencias. La primera concierne a países en los que existen entidades públicas que establecen evaluaciones de los programas de posgrado que pueden ser voluntarias y cuyos resultados en caso de ser aprobatorios se expresan en función de la cantidad de años de acreditación. Así, cuantos más años de acreditación logra obtener el programa, se infiere que la evaluación ha sido más favorable que para los programas que han logrado menos años. Por ejemplo, en el caso chileno, para la Comisión Nacional de Acreditación (s/f), el tiempo máximo de vigencia de un programa de posgrado acreditado muy favorablemente es hasta diez años. La acreditación de un programa de posgrado genera beneficios para los alumnos a través del acceso a fondos concursables de becas con financiamiento estatal. En Chile ningún doctorado en educación ha sido acreditado con el máximo de años, siendo en la actualidad el único doctorado en educación acreditado el de la Pontificia Universidad Católica de Chile, con vigencia por cinco años.

La segunda experiencia es la de aquellos países que han establecido políticas de evaluación de sus posgrados donde sea en forma voluntaria u obliga- toria; los resultados no se miden solo en función de los años de vigencia de la acreditación sino en niveles. Brasil, México y Argentina son los países que han desarrollado en la región esta modalidad evaluativa y en donde el primero tiene un mayor recorrido. En otros países fuera de la región latinoamericana, como en España, se ha desarrollado desde la década anterior un programa que otorga un estatus distintivo para los doctorados que logran cumplir con los requisitos de calidad exigidos, con el nombre de "Mención de Calidad del Doctorado" (Buela-Casal y Castro, 2008).

En Brasil, la entidad responsable de las evaluaciones de posgrado es la Coordinación de Perfeccionamiento del Personal de la Educación Superior (CAPES) con antecedentes desde 1976 y reconocida como Fundación Pública desde 1992. La evaluación es obligatoria y trienal, con base en una calificación de 1 a 7, para lo cual CAPES (2006) determina los criterios e instrumentos de evaluación más precisos para cada área disciplinaria; la evaluación en el caso de la educación se orienta según los siguientes porcentajes: a) cuerpo docente permanente (30\%); b) cuerpo de alumnos, tesis y disertaciones (30\%); c) producción intelectual (30\%); y d) inserción social (10 \%). Existe una segunda evaluación destinada solo para otorgar las calificaciones superiores de nivel 6-7, por considerarse programas de alto desempeño y liderazgo nacional, los cuales son, además, beneficiados por el Programa de Excelencia Académica de la CAPES (PROEX), que les permite aprovechar recursos para financiar becas a los alumnos, publicaciones de los profesores, participación en eventos académicos y equipamiento (CAPES, $s / f$ ).

En el contexto de México, la evaluación es dirigida por el Programa Nacional de Posgrados de Calidad (PNPC) -con ese nombre desde 2006 y con antecedentes desde 1991- como una instancia de gestión entre la Subsecretaría de Educación Superior y el Consejo Nacional de Ciencia y Tecnología (CONACyT). Luego del proceso de evaluación voluntaria, los programas son clasificados en cuatro categorías: 
a) competencia internacional: que incluye aquellos que destacan por sus convenios internacionales que les permite la movilidad de estudiantes y profesores, la codirección de tesis y proyectos de investigación conjuntos; b) consolidado: son así clasificados los programas con un reconocimiento nacional por la pertinencia e impacto en la formación de recursos humanos de alto nivel, en la productividad académica y en la colaboración con otros sectores de la sociedad; c) en desarrollo: son los que mantienen una prospección académica positiva sustentada en su plan de mejora y en las metas factibles de alcanzar a mediano plazo; y finalmente, los llamados de "reciente creación". Los programas de posgrado que son evaluados por el PNPC obtienen como beneficios el otorgamiento de becas para sus alumnos y fondos para estudios de posdoctorado y sabáticos para los docentes (PNPCa s/f). Otra política que refuerza más sistémicamente los posgrados de excelencia en este país es la existencia del Programa de Mejoramiento del Profesorado (PROMEP s/f) que beca a profesores de universidades públicas para realizar estudios en posgrados de alta calidad.

La entidad encargada de las evaluaciones en Argentina es la Comisión Nacional de Evaluación y Acreditación Universitaria (CONEAU), instancia dependiente del Ministerio de Educación, con antecedentes desde inicios de la década de 1990. La CONEAU establece una clasificación, después del proceso de evaluación voluntario, en tres niveles: A: excelentes; B: muy buenas; C: buenas; a los programas nuevos se les acredita por tres años y a los demás por seis años. Solo los postulantes a los posgrados acreditados por el CONEAU son beneficiados para becas gestionadas por el Consejo Nacional de Investigaciones Científicas y Técnicas (CONICET s/fa).

A nivel de accesibilidad a la información de los portales oficiales de las entidades responsables de dichas evaluaciones, en el caso de Brasil y Argentina se puede acceder muy directamente a las actas de acreditación de las universidades, en las cuales se explicita la evaluación realizada, mientras que en el caso de México solo se registra el listado de programas evaluados.

Como se observa en la Tabla 2, en los tres países existen entre 1 y 3 doctorados que ocupan los niveles más altos de calificación y, del total de doctorados en educación, representan para el caso de Brasil y México un porcentaje reducido que no llega a $10 \%$; cuestión diferente para el caso de Argentina, en donde sus doctorados con la mejor calificación representa un porcentaje mayor.

\section{Tabla 2. Evaluación de doctorados en educación en tres países}

\begin{tabular}{|c|c|c|}
\hline Brasil & México & Argentina \\
\hline (a) Total: 45 & (b)Total: 14 & (c) Total: 9 \\
\hline $\begin{array}{c}\text { Nivel 7:3 } \\
\text { (6\%) } \\
\text { Nivel 6:5 } \\
(11 \%) \\
\text { Nivel 5: } 13 \\
(28 \%) \\
\text { Nivel 4: } 24 \\
\text { (53\%) } \\
\text { Niveles 1-2. }\end{array}$ & $\begin{array}{l}\text { Nivel "competencia } \\
\text { Internacional": } 1 \text { (7\%) } \\
\text { Nivel "consolidados": } \\
4 \text { (28 \%) } \\
\text { Nivel "en desarrollo": } \\
7 \text { (50 \%) } \\
\text { Nivel "reciente creación: } \\
2 \text { (14\%) }\end{array}$ & $\begin{array}{l}\text { Nivel A: } 2 \\
(22 \%) \\
\text { Nivel B: } 1 \\
\text { (11\%) } \\
\text { Nivel C: } 6 \\
\text { (66\%) }\end{array}$ \\
\hline
\end{tabular}

\section{Fuentes:}

(a) CAPES. Planilhas comparativas da Avaliação Trienal 2010. http://www.capes.gov.br/component/content/article/44avaliacao/4355-planilhas-comparativas-da-avaliacao-trienal-2010

(b) PNPC Programas vigentes 2012 http://www.conacyt.gob. $\mathrm{mx} /$ FormacionCapitalHumano/Documents/PNPC/Listado_ PNPC 2012.pdf

(c) CONEAU, Buscador por universidad vigentes a 2012. http:// www.coneau.edu.ar/buscadorPosgradolll/

En el contexto brasileño son 3 los doctorados en educación de los 112 doctorados de todas las disciplinas de nivel 7 (CAPES, 2010); en México, de los 55 doctorados de diversas áreas disciplinarias calificados de competencia internacional por el PNPC, solo uno está explícitamente relacionado con educación (CONACYT, s/f), y en el caso de Argentina son dos entre más de 80 los clasificados con el nivel A (CONEAU, 
s/f). En los tres casos, los doctorados en educación de mejor calificación representan menos de $3 \%$ del total de programas similares.

Los tres programas de doctorado en educación en Brasil pertenecen a la Pontificia Universidad Católica - Rio de Janeiro (PUC-Rio), la Universidad Estadual de Rio de Janeiro (UERJ) y la Universidad Federal de Minas Gerais (UFMG). Las dos últimas son universidades públicas mientras que la primera es de gestión privada. De las tres, el doctorado de la PUC-Rio es de mayor antigüedad y la más reciente es el programa de la UERJ; en todos los casos los doctorados se iniciaron entre 11 y 23 años después de la creación de las maestrías en educación, las cuales también han sido acreditadas con la máxima calificación y ambas son presentadas orgánicamente como "programas de posgrado". En el caso de la UERJ su doctorado en educación es el único programa de posgrado de nivel 7; en la PUC-Rio el nivel más alto es compartido con otros dos doctorados (de ingeniería e informática), y en el de la UFMG hay otros siete doctorados que también han sido clasificados en el nivel más alto, relacionados con las ciencias biológicas, astronomía, computación, demografía, ingeniería y uno del campo de la literatura. Estos tres doctorados pertenecen a universidades situadas entre las 35 mejores de América Latina y el Caribe, según el Ranking Iberoamericano de Universidades SIR-2012, que mide diferentes indicadores relacionados con la producción científica de un total de 1.254 universidades de lberoamérica (Scimago, 2012).

El doctorado de México es desarrollado por el Centro de Investigación y de Estudios Avanzados del Instituto Politécnico Nacional (CINVESTAV), institución pública creada a inicios de la década de 1960 como una institución especializada en la investigación en diversos campos y que además ofrece programas de maestría y doctorado. La unidad encargada de la orientación académica del posgrado en educación es el Departamento de Investigaciones Educativas (DIE) fundado en 1971, iniciándose cuatro años después el Programa de Maestría en Ciencias con Especialidad en Investigación Educativa -actualmente acreditada al nivel de "consolidada"- y en 1993 se inicia el doctorado con la misma denominación que la maestría. El mismo DIE ofrece otra maestría y doctorado en ciencias con especialidad en matemática educativa, la primera reconocida con el nivel de "competencia internacional" y la segunda con el nivel de "consolidado". En total, el CINVESTAV cuenta con 27 maestrías y doctorados de "competencia internacional", ubicándose como la institución con más posgrados clasificados en ese nivel (PNPC 2012). Según el Ranking lberoamericano de Universidades SIR-2012 (Scimago, 2012), el CINVESTAV está situado en el puesto 11 dentro de las universidades de América Latina y el Caribe.

Los programas de doctorado de Argentina pertenecen a dos universidades públicas con una diferencia muy pronunciada respecto a sus años de creación. En efecto, el Doctorado en Ciencias de la Educación de la Universidad Nacional de Córdoba (UNCordoba), ubicado en la Facultad de Filosofía y Humanidades, se inició hacia fines de la década de 1960 mientras que el Doctorado en Educación de la Universidad Nacional de Cuyo (UNCuyo), perteneciente a la Facultad de Filosofía y Letras, es creado a mediados de la década de 2000 , es decir, más de 30 años de diferencia entre ambos. En la UNCordoba existe una maestría en pedagogía ofrecida por la misma Facultad en la que se encuentra el Doctorado; en dicha universidad existen más de 20 programas de posgrado (maestría-doctorados) calificados con el nivel A. La UNCuyo cuenta solo con una maestría cercana al campo de la educación ofertada por la misma Facultad, y en cambio, existe otro doctorado denominado "ciencias de la educación" con calificación C. En esta última universidad más de 15 programas de posgrado se encuentran en el nivel A. Un aspecto importante del doctorado en educación de la UNCuyo es que este se desarrolla en convenio con la Université de Rouen (Francia) y el Centre de Recherche Sur la Formation (CNAM, Francia). La UNCordoba se ubica en el puesto 29 y la UNCuyo en el 
94 en la clasificación del Ranking Iberoamericano de Universidades SIR-2012 (Scimago, 2012) en el ámbito de las universidades latinoamericanas (Tabla 3).

Tabla 3. Los doctorados en educación de excelencia en tres países de América Latina

\begin{tabular}{|c|c|c|}
\hline Brasil & México & Argentina \\
\hline $\begin{array}{l}\text { (a) PUCP-Rio: } \\
\text { Doctorado en } \\
\text { Educación (1976) } \\
\text { (b) UFMG: } \\
\text { Doctorado en } \\
\text { Educación (1991) } \\
\text { (c) UERJ: } \\
\text { Doctorado en } \\
\text { Educación (2002) }\end{array}$ & $\begin{array}{l}\text { (d) CINVESTAV: } \\
\text { Doctorado en } \\
\text { ciencias con } \\
\text { especialidad en } \\
\text { investigaciones } \\
\text { educativas (1993). }\end{array}$ & $\begin{array}{l}\text { (e ) UNCórdoba: } \\
\text { Doctorado en } \\
\text { Ciencias de la } \\
\text { Educación (1968) } \\
\text { (f) UNCuyo: } \\
\text { Doctorado en } \\
\text { Educación (2004) }\end{array}$ \\
\hline
\end{tabular}

Fuentes:

(a) CAPES (2010). Ficha de Avaliação do Programa PUC-Rio. Recuperado de: http://trienal.capes.gov.br/wp-content/ uploads/2010/12/fichas/31005012001Po.pdf

(b) CAPES (2010). Ficha de Avaliação do Programa UFMG. Recuperado de: http://trienal.capes.gov.br/wp-content/ uploads/2010/12/fichas/32001010001P7.pdf

(c) CAPES (2010). Ficha de Avaliação do Programa UERJ. Recuperado de: http://trienal.capes.gov.br/wp-content/uploads/2010/12/ fichas/31004016006P5.pdf

(d) DIE Portal institucional. http://www.cinvestav.mx/Departamentos/InvestigacionesEducativas \%28SedeSur \%29.aspx

(e) CONEAU (2007). Documento de acreditación. http://www. coneau.gov.ar/archivos/resoluciones/Res447-07C4316.pdf

(f) CONEAU (2007). Documento de acreditación. http://www. coneau.gov.ar/archivos/resoluciones/Res448-07C4322.pdf

En los casos de Brasil y México, la articulación entre maestría y doctorado parece más articulada como programas de posgrado y secuenciada históricamente, en la medida en que primero se establecieron las maestrías y entre una a dos décadas después se iniciaron los doctorados.

\section{Líneas de investigación en los doctorados en educación de excelencia}

Esta parte está centrada en sistematizar uno de los aspectos más importantes de los doctorados y que atañe a su función estratégica para la producción de conocimientos. Para ello presentaremos, en primer lugar, las formas como son legitimadas las líneas de investigación en los discursos y procedimientos de los posgrados, exceptuando los programas de Argentina por no aportar información explícita en sus portales respectivos (UNCuyo s/f; UNCordoba s/f).

Es en los posgrados de Brasil donde la referencia a las líneas de investigación es más explícita y articuladora. En el reglamento del posgrado PUCP-Rio (2002) se enfatiza la pertenencia de los profesores del programa a las líneas:

Art. $3^{\circ}$. Os Programa de Pós-Graduação deverão contar comum corpo de Professores portadores de título de doutor, ou equivalente, que desenvolverão pesquisas dentro de linhas prioritárias, previamente definidas, na área de conhecimento em que se concentrar o Programa.

En el portal institucional del programa de posgrado en educación la UERJ (PROPED, s/f) se subraya que:

O ProPEd articula-se em torno de Linhas de Pesquisa que evidenciam seu compromisso pedagógico e sócio-político.

O Programa garante a articulação entre estas Linhas de Pesquisa, proporcionando o foco comumna produção e socialização de conhecimentos no campo educacional referenciados no interesse público e na crítica aos processos de exclusão.

Igualmente encontramos esta presencia transversal de las líneas de investigación desde el proceso de admisión de los candidatos al doctorado en el portal del posgrado de la UFMG:

O currículo do Curso de Doutorado em Educação prevê disciplinas, seminários de pesquisa e atividades, constituidos no contexto da linha de pesquisa para a qual o candidato é admitido. O Colegiado do Programa é a instância de discussão e acompanhamento da trajetória dos alunos de 
ISSN 0123-1294 | Educ.Educ. Vol. 16. No. 3 | Septiembre-diciembre de 2013 | pp. 433-451.

Universidad de La Sabana | Facultad de Educación

Doutorado, coordenando atividades e disciplinas que sejam de interesse comum aos alunos inseridos em diferentes linhas de pesquisa.

En el caso del doctorado mexicano, en la web de presentación del Departamento encargado del doctorado, el DIE, se alude al concepto de líneas de investigación al reconstruir la historia de dicha unidad académica y en una parte más específica se menciona que: "Los profesores del programa desarrollan líneas de investigación reconocidas a nivel nacional y cuentan con sólidos contactos profesionales de orden internacional" (DIE, s/f). En el mismo portal se utiliza el concepto de "Líneas de Generación y Aplicación de Conocimiento (LGAC)" para dar cuenta de las grandes líneas de investigación que definen las pertenencias temáticas de los docentes. Esta denominación de LGAC no es exclusiva del DIE sino que forma parte de la cultura institucional de los posgrados en México, la cual es definida en el portal del PNPCb (s/f):

Una línea de generación y/o aplicación del conocimiento (LGAC) es un campo temático en la cual confluyen las trayectorias de investigaciones de los profesores que integran el núcleo académico básico de un programa de posgrado $y$ el trabajo de los estudiantes desde una perspectiva sistémica de generación de nuevo conocimiento, o aplicación. Las LGAC definen la naturaleza del programa.

En la Tabla 4 se muestran las líneas de investigación de los doctorados de Brasil y México, las cuales son más explicadas en las páginas web de los primeros programas que en el segundo. Observamos la presencia de cuatro a nueve líneas articuladoras en los doctorados, de cuyos discursos de presentación podemos esbozar las siguientes comparaciones.

Desde el punto de vista de los enfoques multidisciplinarios, están presentes más transversalmente en los programas: a) el enfoque histórico de la educación; b) el enfoque sociocultural de la educación, alimentados, como se expresa en algunas líneas desde la antropología y la sociología; c) los que remiten al enfoque curricular y didáctico. En forma más particular, hay líneas que revelan enfoques multidisciplinarios muy fusionados como la de "Aprendizaje y Desarrollo", que se propone investigar los "procesos sociopsicolingüísticos de la lectoescritura".

En cuanto a las opciones teóricas más especificas, en algunos casos esta queda explicitada en el propio nombre de la línea, como es el caso de "Psicología, Psicoanálisis y Educación"; siendo muy evidente la opción por el psicoanálisis como matriz fundamental desde la cual la línea se desarrolla teóricamente. Otra similar es la línea "Cotidianos, redes educativas y procesos culturales"; en su discurso de presentación no solo se incluyen conceptos clave sino también diferentes autores que representan un rango de aproximaciones teóricas legitimado por los investigadores de dicha línea.

Por otro lado, desde la perspectiva de los sujetos de referencia se explicitan en al menos una línea en cada programa de doctorado, al sujeto docente como objeto de estudio, focalizando en aspectos relativos a su historia, su formación, condiciones de trabajo, práctica profesional y desarrollo profesional. En otras líneas se alude a otros sujetos en referencia a su periodo etario (infancia, juventud) o como sujeto social (trabajadores, migrantes, familia). También hay líneas que utilizan el término en general "sujeto" en sus denominaciones.

Este mapeo global de las líneas de investigación permite distinguir las especializaciones que han venido acumulando dichos programas y que constituyen sus fortalezas y capacidades para configurar campos de estudios desde los cuales se articulan la producción docente y las tesis de los alumnos, como otras actividades académicas.

\section{Los docentes-investigadores en los programas de excelencia en educación}

Las 23 líneas de investigación de los cuatro doctorados seleccionados -exceptuando los de Argentina-están conformados por 160 investigadores; 
Tabla 4. Lineas de investigación en cuatro programas de educación de excelencia

\begin{tabular}{|c|c|c|c|}
\hline PUC-Rio (a) & UERJ (b) & UFMG (c) & CINVESTAV (d) \\
\hline $\begin{array}{l}\text { Historia de las ideas e } \\
\text { instituciones educacionales } \\
\text { (ideas educacionales en Brasil } \\
\text { desde la perspectiva filosófica e } \\
\text { histórica) }\end{array}$ & $\begin{array}{l}\text { Instituciones, prácticas } \\
\text { educativas e historia (historia de } \\
\text { la profesión docente, de la lecto- } \\
\text { escritura, de manuales escolares, } \\
\text { de las instituciones) }\end{array}$ & $\begin{array}{l}\text { Historia de la educación (historia } \\
\text { de educación y género, etnia, } \\
\text { generación, clase social, escuela, } \\
\text { currículo) }\end{array}$ & $\begin{array}{l}\text { Historia de la educación y del } \\
\text { conocimiento (historia social y } \\
\text { cultural de la educación, ciencias } \\
\text { y medicina) }\end{array}$ \\
\hline $\begin{array}{l}\text { Procesos culturales, instancias } \\
\text { de socialización y educación } \\
\text { (infancia, juventud y cultura; } \\
\text { multiculturalismo y educación; } \\
\text { relación de agentes sociales con } \\
\text { cultura letrada) }\end{array}$ & $\begin{array}{l}\text { Cotidianos, Redes Educativas y } \\
\text { Procesos Culturales (redes de } \\
\text { conocimientos; identidades; } \\
\text { artefactos culturales y currículo; } \\
\text { discriminación; lenguajes en lo } \\
\text { cotidiano) }\end{array}$ & $\begin{array}{l}\text { Educación, cultura, movimientos } \\
\text { sociales y acciones colectivas } \\
\text { (educación y diversidad étnico- } \\
\text { racial; educación jóvenes-adultos; } \\
\text { educación y violencia) }\end{array}$ & $\begin{array}{l}\text { Estudios socioculturales sobre } \\
\text { la escuela, aula y comunidad } \\
\text { (TICs dentro y fuera de la escuela; } \\
\text { familias, migrantes, lengua } \\
\text { escrita y oral) }\end{array}$ \\
\hline $\begin{array}{l}\text { Educación, relaciones sociales } \\
\text { y construcción democrática } \\
\text { (democratización de la } \\
\text { educación; desigualdades } \\
\text { sociales, equidad) }\end{array}$ & $\begin{array}{l}\text { Curriculum: sujetos, } \\
\text { conocimiento y cultura (políticas } \\
\text { y prácticas curriculares en } \\
\text { educación básica y formación } \\
\text { docente) }\end{array}$ & $\begin{array}{l}\text { Educación escolar: instituciones, } \\
\text { sujetos y currículos (familia- } \\
\text { escuela; trayectorias escolares } \\
\text { y desigualdades; condición, } \\
\text { formación e identidad docente; } \\
\text { artefactos culturales y practicas } \\
\text { educativas; aula) }\end{array}$ & $\begin{array}{l}\text { Didácticas especializadas: lengua, } \\
\text { matemáticas, ciencias naturales } \\
\text { y ciencias sociales (discurso } \\
\text { científico escolar; percepción } \\
\text { y desarrollo profesional de } \\
\text { docentes de ciencias) }\end{array}$ \\
\hline \multirow[t]{6}{*}{$\begin{array}{l}\text { Formación de profesores: } \\
\text { tendencias y dilemas (identidad } \\
\text { y socialización profesional; } \\
\text { trabajo y práctica docente, saber } \\
\text { docente) }\end{array}$} & $\begin{array}{l}\text { Educación Inclusiva y Procesos } \\
\text { Educativos (tolerancia y } \\
\text { educación; fracaso escolar) }\end{array}$ & $\begin{array}{l}\text { Educación y ciencias (enseñanza- } \\
\text { aprendizaje-evaluación de } \\
\text { ciencias; prácticas discursivas } \\
\text { en aula; procesos cognitivos- } \\
\text { afectivos; políticas) }\end{array}$ & $\begin{array}{l}\text { Políticas e instituciones de } \\
\text { educación, ciencia y tecnología } \\
\text { (ed. superior; sociología de invest. } \\
\text { científica) }\end{array}$ \\
\hline & $\begin{array}{l}\text { Infancia, Juventud y Educación } \\
\text { (como actores sociales; políticas } \\
\text { para su ciudadanía) }\end{array}$ & $\begin{array}{l}\text { Educación matemática (prácticas } \\
\text { pedagógicas; formación docente) }\end{array}$ & $\begin{array}{l}\text { Aprendizaje y desarrollo } \\
\text { (psicogénesis de la } \\
\text { lengua escrita; procesos } \\
\text { sociopsicolingüísticos de lecto- } \\
\text { escritura; didáctica de la lengua) }\end{array}$ \\
\hline & & $\begin{array}{l}\text { Educación y lenguaje (lengua } \\
\text { portuguesa; alfabetización; } \\
\text { formación docente) }\end{array}$ & \\
\hline & & $\begin{array}{l}\text { Política, trabajo y formación } \\
\text { humana (trabajadores, } \\
\text { mov. sociales y sus procesos } \\
\text { educativos) }\end{array}$ & \\
\hline & & $\begin{array}{l}\text { Políticas públicas de educación: } \\
\text { concepción, implementación } \\
\text { y evaluación (fundamentos } \\
\text { y evaluación de las políticas; } \\
\text { estudios comparativos; } \\
\text { organización del trabajo escolar) }\end{array}$ & \\
\hline & & $\begin{array}{l}\text { Psicología, psicoanálisis y } \\
\text { educación (psicoanálisis } \\
\text { aplicados a problemas } \\
\text { educativos; éxito, fracaso e } \\
\text { inclusión escolar; lenguaje, } \\
\text { cultura y cognición en aula) }\end{array}$ & \\
\hline
\end{tabular}

Fuentes:

(a) Web de PUC-Rio, Departamento de Educación-Posgraduación. http://www.edu.puc-rio.br/pos_graduacao_linhaspesq.shtml

(b) WEB de UERJ-PROPED. http://www.proped.pro.br/

(c) WEB UFMG - Programa de posgraduación. http://www.posgrad.fae.ufmg.br/site/index.php/Linhas-de-Pesquisa

(d) DIE. Líneas de Generación y Aplicación de Conocimiento (LGAC). http://www.die.cinvestav.mx/Programasdeposgrado/Doctorado/Con vocatoria2013/2013ConvocaDocLGAC.aspx 
a cada línea pertenecen entre 2 y 13 investigadores, esto hace una media de casi 7 docentes, siendo la línea de mayor número de integrantes la de "Educación escolar: instituciones, sujetos y currículos" (UFMG), y la de menor cantidad de integrantes la de "Didácticas especializadas: lengua, matemáticas, ciencias naturales y ciencias sociales" (CINVESTAV).

Un rasgo importante de los profesores de estos programas es que poco más de un tercio de ellos han sido acreditados como investigadores por los sistemas de sus países encargados de promover políticas de reconocimiento y estímulos a los profesores que logran ser admitidos en dichos sistemas.

En Brasil el CNPq ha implantado esta política desde 1976 y permite a los investigadores postular voluntariamente a este sistema mediante el cual son evaluados periódicamente y denominados como "Bolsista de Produtividade em Pesquisa". Un requisito básico para ello es ser doctor o tener un perfil científico equivalente y estar vinculado a instituciones de investigación y enseñanza. Se evalúa para ello la relevancia, originalidad y repercusión de la producción científica del candidato, su participación en proyectos y redes de investigación, su inserción internacional, su labor como editor científico, entre otros. El candidato es clasificado por categoría y niveles. En el primer caso, la categoría 1 es asignada a los que tienen 8 años como mínimo de haber obtenido el grado de doctor, y la categoría 2, para aquellos que tienen mínimo 3 años. Solo para los de la categoría 1 se aplica la clasificación en niveles (A, $B, C, D)$, siendo el nivel A para los investigadores de mayor excelencia académica continua de producción científica, formación de recursos humanos y liderazgo en grupos de investigación consolidados (CNPq, 2006). En los tres doctorados de educación seleccionados del Brasil, entre 36 y $42 \%$ de sus profesores tiene el estatus de "bolsistas de Produtividade em Pesquisa" en las categorías 1 y 2; y hay mínimamente un profesor de nivel $1 \mathrm{~A}$ en cada uno de los programas.
En México, el Consejo Nacional de Ciencia y Tecnología (CONACYT) es el ente regulador de las políticas del Sistema Nacional de Investigadores (SIN), creado desde 1984 para evaluar a los que voluntariamente desean postular al sistema y reconocerlos con la distinción de "Investigador Nacional", en función del cual se aplican diversas políticas de incentivos. Los aceptados con esa distinción son clasificados en tres niveles, siendo el tercero el de mayor valoración por haber logrado un reconocimiento nacional e internacional a su actividad científica o tecnológica y haber realizado una destacada labor en la formación de recursos humanos de alto nivel para el país (CONACYT, 2012). El $90 \%$ de los profesores del doctorado del CINVESTAV son parte del SIN y cuatro de ellos pertenecen al nivel más alto. Los 20 docentes investigadores de este programa son parte de los aproximadamente 467 investigadores educacionales pertenecientes al SIN que Colina (2011) identificó en su estudio (Tabla 5).

En el caso argentino aunque existe la carrera del investigador científico y tecnológico gestionada desde el CONICET (s/f, b) y un Programa de Incentivos a Docentes Investigadores que permite clasificarlos en cinco niveles por la Secretaría de políticas universitarias del Ministerio de Educación de Argentina (s/f), no encontramos datos en las páginas electrónicas de los dos doctorados que seleccionamos para este estudio que permita identificar a sus docentes en función de dichas clasificaciones.

Otro rasgo que evidencia el perfil del docente de dichos programas es el relacionado con su producción intelectual. Para ello, como se observa en la Tabla 6, en los últimos cinco años el número de artículos en revistas académicas oscila entre 53 (CINVESTAV) y 692 (UFMG), los que a la vez representan los extremos en cantidad mínima y máxima de docentes. En los programas brasileños los docentes tienden a publicar aproximadamente 1.5 artículos, mientras que en el caso mexicano está por debajo de 0.5; sin embargo, la producción de este último programa es la más alta en 
porcentaje de artículos publicados en idiomas extranjeros, llegando a constituir la tercera parte de su producción, muy distante de los artículos publicados en idiomas extranjeros distintos del portugués por los docentes de Brasil. Otro rasgo importante es el referido a las coautorías en la cual se evidencia una tendencia en todos los programas -entre uno y dos tercios- como forma de publicación entre dos o más autores y que puede estar revelando las redes que moviliza cada docente dentro y fuera de su universidad y país para su producción académica.

Tabla 5. Profesores de los programas en educación de excelencia

\begin{tabular}{|c|c|c|c|c|}
\hline & PUC-Rio (a) & UERJ (b) & UFMG (c) & CINVESTAV $(\mathrm{d})$ \\
\hline Número de profesores & 21 & 33 & 84 & 22 \\
\hline $\begin{array}{l}\text { Número y porcentaje de } \\
\text { acreditados* y número } \\
\text { con nivel mayor de } \\
\text { acreditación }\end{array}$ & $\begin{array}{c}9(42 \%) \\
\text { (3 nivel 1 A) }\end{array}$ & $\begin{array}{c}13(39 \%) \\
(1 \text { nivel } 1 \text { A) }\end{array}$ & $\begin{array}{c}31(36 \%) \\
(3 \text { nivel } 1 \text { A) }\end{array}$ & $\begin{array}{l}20(90 \%) \\
(4 \text { nivel III) }\end{array}$ \\
\hline
\end{tabular}

Fuentes:

(a) Departamento de Investigación - Líneas de investigación. http://www.edu.puc-rio.br/pos_graduacao_linhaspesq.shtml

(b) Proped líneas de investigación. http://www.proped.pro.br/

(c) Programa de posgraduación - profesores. http://www.posgrad.fae.ufmg.br/site/index.php/Pessoas/Professores

(d) DIE-Personal académico. http://www.die.cinvestav.mx/Personalacad \%C3 \%Agmico.aspx

*Nota: acreditados como investigadores por la CNPq en Brasil y por el SIN en México.

Tabla 6. Artículos publicados por profesores en revistas académicas

\begin{tabular}{|c|c|c|c|c|}
\hline & PUC - Rio (a) & UERJ (b) & UFMG (c) & CINVESTAV (d) \\
\hline Total 2007-2012 & 148 & 264 & 692 & 53 \\
\hline Promedio anual & 29.6 & 52.8 & 138.4 & 10.6 \\
\hline Promedio anual por docente & 1.4 & 1.6 & 1.6 & 0.4 \\
\hline En otros idiomas & $10(6 \%)$ & $26(9 \%)$ & $86(12 \%)$ & $18(33 \%)$ \\
\hline Coautorías & $76(51 \%)$ & $135(51 \%)$ & $456(65 \%)$ & $19(35 \%)$ \\
\hline
\end{tabular}

\section{Fuentes:}

(a) Departamento de Investigación - Líneas de investigación. http://www.edu.puc-rio.br/pos_graduacao_linhaspesq.shtml

(b) Proped líneas de investigación. http://www.proped.pro.br/

(c) Programa posgrado - Líneas de investigación. http://www.posgrad.fae.ufmg.br/site/index.php/Linhas-de-Pesquisa

(d) DIE-CINVESTAV. http://www.die.cinvestav.mx/Personalacad \%C3 \%Agmico.aspx 


\section{Conclusiones}

Los doctorados de excelencia analizados en este trabajo son programas que forman parte del campo científico en sus respectivos países logrando posicionarse con un alto nivel de reconocimiento académico. En ese sentido, dichos doctorados contribuyen a la legitimidad científica de la educación como área de conocimiento relevante ante la cual es posible organizar comunidades académicas comprometidas con la producción de conocimientos y formación de investigadores para dicha área.

Desde la perspectiva alimentada por Bourdieu (2002) y otros (Putnam, 2009; Gunter, 2002; Díaz, 1993) para el análisis del campo intelectual como campo de poder -en el cual se libran luchas por recursos y hegemonías desde actores con diferentes trayectorias socio-históricas-, podemos interpretar que la presencia de los doctorados en educación representan un proceso de flexibilización a la hegemonía tradicional de considerar científicas mayormente a las áreas de las llamadas ciencias duras o sociales con fuertes tradiciones metodológicas. La educación como área de conocimiento y las comunidades académicas involucradas en su estudio, como la perteneciente a los doctorados, son reconocidas al mismo nivel de calidad de producción y formación que las ciencias biológicas, las ingenierías o la astronomía, como ocurre en el caso de la UFMG y en el CINVESTAV. Más aún, en contextos como el de la UERJ el único posgrado reconocido del más alto nivel es el de Educación.

En los doctorados examinados, como en CINVESTAV, UMFG y UNCordoba es más claro que ellos se ubican en contextos institucionales de alta calidad, en donde la cultura de excelencia ha sido reconocida a través de una cantidad apreciable de maestrías y doctorados clasificados en los más altos niveles por sus sistemas de evaluación nacional y por los rankings internacionales como el de SCIMAGO que sitúa dichas universidades en el quinto superior a nivel latinoamericano. En esos ámbitos, los doctorados en educación pueden estar representando el papel de reforzadores de los patrones de excelencia de la cultura organizacional de la misma universidad. Estos patrones son producto de las prácticas de gestión del conocimiento internas al programa, a la universidad y las políticas de evaluación desde el Estado hacia los posgrados. Estas tres interacciones son fundamentales para comprender el camino de aprendizajes de los doctorados de excelencia como procesos hacia adentro y hacia afuera, donde el "Estado evaluador" (Betancur, 1996) manifiesta su presencia regulatoria justificando su rol en la educación superior sustentado en el discurso de la calidad educativa.

Las líneas de investigación de los programas de doctorado en educación representan territorios simbólicos y epistemológicos que permiten la construcción de una identidad intelectual, institucional y profesional en los docentes en tanto investigadores (Colina, 2008), en la medida en que desde dichas líneas los docentes construyen sentidos de pertenencia y articulación entre saberes multidisciplinarios y formativos que trasciende el corto plazo. Los enfoques históricos, socioculturales, curriculares y otros presentes en los discursos que sustentan las líneas de investigación, revelan que los enfoques multidisciplinarios y multirreferenciales son un rasgo fundamental en la forma de construir conocimiento desde dichos doctorados. Estas líneas delimitan, además, diferentes énfasis en la manera de relacionar al menos cinco aspectos clave en sus conceptualizaciones: prácticas, procesos, contextos, sujetos y mediaciones. Cada uno de estos conceptos vertebra las perspectivas conceptuales con las cuales el campo educativo es representado en tanto objeto de estudio imposible de ser traducido sin un andamiaje semántico que delimite fronteras flexibles para transitar en el camino del conocimiento. El estudio de estas líneas, los grupos y proyectos de investigación que las alimentan son la columna vertebral de dichos programas que merecen mayores estudios sobre su gestión, productividad e impacto.

La información que nos aporta este estudio sobre la calidad de los docentes investigadores de 
los programas de doctorado requiere una contextualización mayor para una discusión más histórica sobre la trayectoria de sus estatus académicos y productividad intelectual. En efecto, para los países estudiados, tienen una importancia fundamental las políticas estatales de promoción y acreditación de investigadores en general y, en especial, en el campo de la educación. Sin duda, Brasil y México han desarrollado una trayectoria de mayor continuidad en dichas políticas que fue impactando aun con sus problemas particulares en el desarrollo profesional de los docentes-investigadores adscritos a los doctorados. En Brasil desde la década de 1950 se crearon los Centros Regionales de Estudios Educacionales en diversos estados del Brasil vinculados al Instituto Nacional de Estudios Pedagógicos fundado en 1938 (Ferreira, 2008). México también cuenta con antecedentes de políticas de investigación educativas que datan de 1936 con la creación del Instituto Nacional de Psicopedagogía (INP), fundado por la Secretaría de Educación Pública y convertido en 1970 en el Instituto Nacional de Investigación Educativa (INIE) (Colina, 2011).

Otro factor contextual necesario de resaltar es la propia organización independiente de los investigadores educacionales en dichos países como forma de legitimar su profesión, generar proyectos de colaboración intelectual y de influencia en la discusión educativa. En el caso de Brasil, la Asociación de Posgraduación y Pesquisa en Educación (ANPED), fundada en 1976, ha desempeñado un rol sustancial en esa dirección, articulando a sus investigadores en más de 20 grupos de trabajo (Handfas, 2008; Sousa y Bianchetti, 2007). En México, la presencia de investigadores educacionales ha tenido como antecedentes al Centro de Estudios Educativos (CEE) en 1963 y, desde 1993, al Consejo Mexicano de Investigación Educativa (COMIE), que agrupa a cerca de 350 investigadores del país (Flores-Crespo, 2004; Weiss, 2007; Colina, 2011).

Creemos que esta contextualización del campo de la investigación educativa es pertinente para comprender mejor el proceso histórico en el cual se insertan los investigadores que forman parte de los doctorados en educación y que nos sugiere la presencia de "generaciones de investigadores" marcados por periodos diferentes de la propia evolución de los posgrados y políticas de investigación de sus países.

Por último, consideramos necesario seguir explorando en el perfil de productividad de este tipo de docente-investigador, no solo sobre el volumen, idioma y coautorías, de lo cual aquí hemos dado cuenta de forma parcial al limitarnos a los artículos en revistas, sino también sobre el impacto de dicha producción en la creación de corrientes teóricasmetodológicas de alcance internacional, como la identificada, por ejemplo, por Weiss (2003), sobre la línea de investigación etnográfica desde el DIECINVESTAV para el caso de México; así como en el nivel de incidencia en las propias tesis de doctorado y maestría en educación.

\section{Referencias}

Acuña, O.Y. (2009). Tendencias historiográficas vistas a través de las tesis del doctorado en ciencias de la educación RUDECOLOMBIA (1998-2007). Revista Historia de la Educación Latinoamericana, 12, 79-95.

Buela-Casal, G. y Castro, A. (2008). Análisis de la evolución de los programas de doctorado con mención de calidad en las universidades españolas y pautas para su mejora. Revista de Investigación en Educación, 5, 49-6o. 
ISSN 0123-1294 | Educ.Educ. Vol. 16. No. 3 | Septiembre-diciembre de 2013 | pp. 433-451.

Universidad de La Sabana | Facultad de Educación

Betancur, V. (1996). El Estado evaluador de la educación superior: pertinencia teórica y modelos. Universidades, 2, 3-11.

Bourdieu, P. (2002). Campo de poder, campo intelectual. Itinerario de un concepto. Argentina: Montressor.

CAPES (2006). Critérios de Avaliação Trienal TriênioAvaliado - 2004 - 2006. Área de Avaliação: EDUCAÇÃO. Recuperado el 14 de abril de 2012 de: http://capes.gov.br/images/stories/download/avaliacao/CA2007_Educacao.pdf

CAPES (2010). Resultados Finais. Recuperado el 28 de enero de 2011 de: http://trienal.capes.gov.br/?page_id=100

CAPES (s/f). Programa de Excelência Acadêmica (Proex). Recuperado el 28 de enero de 2011 de: http://www. capes.gov.br/bolsas/bolsas-no-pais/proex

Cardoso, E.y Cerecedo, M. (2011). Propuesta de indicadores para evaluar la calidad de un programa de posgrado en Educación. REDIE. Revista Electrónica de Investigación Educativa, 13 (2), 68-82.

Colina, A. (2011). El crecimiento del campo de la investigación educativa en México. Un análisis a través de sus agentes. Perfiles Educativos, 23 (132), 10-28.

Colina, A. (2008). Abriendo puertas y saltando obstáculos: la construcción de la identidad del investigador en educación en México. Sinéctica [Revista electrónica], 31. Recuperado el 8 de abril de 2013 de: http://portal. iteso.mx/portal/page/portal/Sinectica/Revista/SIN31Articulooo2

CNA (s/f). Comisión Nacional de Acreditacion-Chile. Recuperado el 12 de julio de 2012 de: http://www.cnachile.cl/

CNPq (2006). Bolsas Individuais no País. Anexo 1. Recuperado el 8 de mayo de 2012 de: http://www.cnpq.br/ web/guest/view/-/journal_content/56_INSTANCE_ooED/10157/100343\#16061

CONACYT (2012). Reglamento Vigente 2013. Recuperado el 22 de enero de 2012 de: http://www.conacyt.gob.mx/ SNI/Documents/Reglamento_2013.pdf

CONACYT (s/f). Padrón del Programa Nacional de Posgrados de Calidad. Sistema de consultas. Recuperado el 22 de enero de 2013 de: http://svrtmp.main.conacyt.mx/ConsultasPNPC/listar_padron.php?prog=\&ref=\&in $\mathrm{st}=\& \mathrm{sgrado}=5 \&$ sorient $=\&$ snivel $=5 \&$ sasni $=\&$ smodal $=\&$ sentidad $=\& p a g=1$

CONICET (s/f, a). Acerca de las becas doctorales y post doctorales del Consejo Nacional de Investigaciones Científicas y Técnicas. Recuperado el 22 de enero de 2013: http://www.conicet.gov.ar/new_scp/noticias. php?id_noticia $=8204 \&$ nota_completa $=$ yes

CONICET (s/f, b). La carrera del investigador científico y tecnológico. Recuperado el 12 de enero de 2013: http:// web.conicet.gov.ar/web/conicet.trabajar.investigador

CONEAU (s/f). Buscador del Portal institucional. Recuperado el 22 de enero de 2013: http://www.coneau.edu.ar/ buscadorPosgradolll/ 
Departamento de Investigaciones Educativas (DIE) (s/f). Portal institucional. Recuperado el 22 de enero de 2013: http://www.cinvestav.mx/Departamentos/InvestigacionesEducativas \%28SedeSur \%29.aspx

DIE (s/f). Convocatoria Doctorado 2013. Doctorado en ciencias en la Especialidad de Investigaciones Educativas. Recuperado el 22 de enero de 2013: http://www.die.cinvestav.mx/Programasdeposgrado/Doctorado/Convocatoria2013.aspx

Díaz, M. (1993). El campo intelectual de la educación. Cali:Textos Universitarios. Universidad del Valle.

Duarte, A. (2010). A produção acadêmica sobre trabalho docente na educação básica no Brasil: 1987-2007. Educar em Revista, 27(1), 101-117.

Fernández, N.; Mollis, M. y Dono, S. (2005). La educación comparada en América Latina: situación y desafíos para su consolidación Académica. Revista Española de Educación Comparada, 11, 161-187.

Fernández, N. (2012). La educación superior en América Latina. Aportes para la construcción de una nueva agenda. Debate universitario [Revista electrónica], 1 (1). Recuperado el 21 de marzo de 2013: http://ppct.caicyt. gov.ar/index.php/debate-universitario/article/viewFile/1603/pdf_1

Ferreira, M. S. (2008). Os Centros de Pesquisas Educacionais do INEP e os estudos em ciências sociais sobre a educação no Brasil. Revista Brasileira de Educação, 1, 38, 279-292.

Flores-Crespo, P. (2004). Conocimiento y política educativa en México. Condiciones políticas y organizativas. Perfiles Educativos, 105-106, 73-101.

Gutiérrez, N. y Barrón, M. C. (2008). Tesis de posgrado en educación en el estado de Morelos. Temas y ámbitos de estudio. Perfiles Educativos, 122, 78-93.

Gusmão, R. (2010). Desarrollo de las capacidades de investigación en ciencias sociales en Brasil. En CICS, Informe Mundial sobre las Ciencias Sociales 2010 (pp.132-136). Paris: UNESCO.

Gunter, H. (2002). Purposes and Positions in the Field of Education Management. Putting Bourdieu to Work. Educational Management \& Administration, 30(1), 7-26.

Handfas, A. (2008). A trajetória do GT Trabalho e Educação da ANPEd: alguns elementos de análise. Trabalho, educação e saúde, 3, 375-398.

Marquis, C.; Spagnolo, F. y Valenti, G. (1998). Desarrollo y acreditación de los posgrados en Argentina, Brasil y México. Textos para una mirada comparativa. Buenos Aires: Ministerio de Cultura y Educación Secretaría de Políticas Universitarias.

Martínez, F. (2001). El doctorado interinstitucional en educación de la UAA. Reflexiones sobre una experiencia. Revista Mexicana de Investigación Educativa, 6 (12), 335-370. 
ISSN 0123-1294 | Educ.Educ. Vol. 16. No. 3 | Septiembre-diciembre de 2013 | pp. 433-451.

Universidad de La Sabana | Facultad de Educación

Ministerio de Educación de Argentina (s/f). Programa de Incentivos a docentes - investigadores. Recuperado el 12 de marzo de 2013: http://portales.educacion.gov.ar/spu/programas/programa-de-incentivos-a-docentes-investigadores/

Moreno, M. G. (2007). Experiencias de formación y formadores en programas de doctorado en educación. Revista Mexicana de Investigación Educativa, 12(33), 561-580.

PNPC (2012). Programa Nacional de Posgrados de Calidad. Programas vigentes 2012. Recuperado el 12 de marzo de 2013: http://www.conacyt.gob.mx/FormacionCapitalHumano/Documents/PNPC/Listado_PNPC_2012.pdf

PNPC (s/f.a). Programa Nacional de Posgrados de Calidad. Recuperado el 22 de enero de 2013: http://www.conacyt.gob.mx/Becas/calidad/Paginas/Becas_ProgramasPosgradosNacionalesCalidad.aspx

PNPC (s/f, b). Sistema de consultas. Recuperado el 12 de marzo de 2013: http://svrtmp.main.conacyt.mx/ConsultasPNPC/ayuda.php

PROMEP (s/f). Programa de Mejoramiento del Profesorado. Recuperado el 22 de enero de 2013: http://promep. sep.gob.mx/presentacion.html

PROPED (s/f). Portal institucional. Recuperado el 12 de enero de 2013: http://www.proped.pro.br/

PUCP-Rio (2002). Regulamento dos cursos de pós-graduação stricto sensu. Recuperado el 12 de enero de 2013: http://www.puc-rio.br/ensinopesq/ccpg/regras/

Putnam, L. (2009). Symbolic Capital and Academic Fields. Management Communication Quarterly, 23 (1), 127-134.

Rama, C. (2006). Los postgrados en América Latina en la sociedad del saber. En IESALC, Informe sobre la educación superior en América Latina y el Caribe. 2000-2005. La metamorfosis de la educación superior (pp.4355). Venezuela: IESALC.

Santos, A. dos, y Azevedo, J. de (2009). A pós-graduação no Brasil, a pesquisa em educação e os estudos sobre a política educacional: os contornos da constituição de um campo acadêmico. Revista Brasileira de Educação, 14(42), 534-550.

SCIMAGO (2012). Ranking Iberoamericano SIR 2012. Recuperado el 12 de enero de 2013: http://www.scimagoir. com/pdf/ranking_iberoamericano_2012.pdf

Sousa, S. Z. y Bianchetti, L. (2007). Pós-graduação e pesquisa em educação no Brasil: o protagonismo da ANPEd. Revista Brasileira de Educação, 12, (36), 389-409.

Torres, M. G. (2011). La tutoría en programas de doctorado. Tensiones tutoriales doctorado en ciencias de la educación RUDECOLOMBIA. Revista Historia de la Educación Latinoamericana, 13 (17), 315-344.

UNCuyo (s/f). Doctorado en Educación. Recuperado el 12 de enero de 2013: http://ffyl.uncu.edu.ar/spip. php?rubriqueg2 
UNCordoba (s/f). Doctorado de Educación. Recuperado el 2 de febrero de 2013: http://www.ffyh.unc.edu.ar/ posgrado/doctorados/doctorado-en-ciencias-de-la-educacion/

Villanueva, E. (2008). Reformas de la educación superior: 25 propuestas para la educación superior en América Latina y el Caribe. En: A. Gazzola, y A. Didriksson (eds.) Tendencias de la Educación Superior en América Latina y el Caribe (pp.241-295). Caracas: IESALC-UNESCO.

Weiss, E. (2007). El desarrollo de la investigación educativa en México, avances y retos. En O. López (coord.) Entre lo emergente y lo posible. Desafíos compartidos en la investigación educativa (pp.131-149). México: Ediciones Pomares, S.A.

Weiss, E. (2003). Diagnósticos, panoramas y estados de conocimiento. En: E. Weiss (coord.), El campo de la Investigación Educativa (pp. 57-64). Mexico: COMIE.

Zaidan, S.; Caldeira, A. M.; Oliveira, B. J.; Silva, P. G. (2011). Pós-Graduação, saberes e formação docente: uma análise das repercussões dos cursos de mestrado e doutorado na prática pedagógica de egressos do Programa de Pós-Graduação da Faculdade de Educação da UFMG (1977-2006). Educação em Revista, 27 (1), 129-160.

Zarur, X. (2008). Integración regional e internacionalización de la educación superior en América latina y el Caribe. En: L. Gazzola y A. Didriksson (eds.) Tendencias de la Educación Superior en América Latina y el Caribe (pp. 179-240). Caracas: IESALC-UNESCO. 
